\title{
Approximate Consistency: Towards Foundations of Approximate Kernel Selection
}

\author{
Lizhong Ding and Shizhong Liao* \\ School of Computer Science and Technology \\ Tianjin University, Tianjin 300072, China \\ szliao@tju.edu.cn
}

\begin{abstract}
Kernel selection is critical to kernel methods. Approximate kernel selection is an emerging approach to alleviating the computational burdens of kernel selection by introducing kernel matrix approximation. Theoretical problems faced by approximate kernel selection are how kernel matrix approximation impacts kernel selection and whether this impact can be ignored for large enough examples. In this paper, we introduce the notion of approximate consistency for kernel matrix approximation algorithm to tackle the theoretical problems and establish the preliminary foundations of approximate kernel selection. By analyzing the approximate consistency of kernel matrix approximation algorithms, we can answer the question that, under what conditions, and how, the approximate kernel selection criterion converges to the accurate one. Taking two kernel selection criteria as examples, we analyze the approximate consistency of Nyström approximation and multilevel circulant matrix approximation. Finally, we empirically verify our theoretical findings.
\end{abstract}

\section{Introduction}

Since learning is ill-posed and data by itself is not sufficient to find the solution [1], some extra assumptions should be made to have a unique solution. The set of assumptions we make to have learning possible is called the inductive bias [21]. Model selection is the process of choosing the inductive bias, which is fundamental to learning. For kernel based learning, model selection involves the selection of the kernel function, which determines the reproducing kernel Hilbert space (RKHS), and the regularization parameter. The selection of regularization parameter has typically been solved by means of cross validation, generalized cross validation [14], theoretical estimation [8] or regularization path [15]. This paper focuses on the kernel selection problem, which is a challenging and central problem in kernel based learning [20].

Kernel selection is to select the optimal kernel in a prescribed kernel set by minimizing some kernel selection criterion that is usually defined via the estimate of the expected error [3]. The estimate can be empirical or theoretical. The $k$-fold cross validation $(\mathrm{CV})$ is a commonly used empirical estimate of the expected error and the extreme form of cross validation, leave-one-out (LOO), gives an almost unbiased estimate of the expected error [6]. However, CV and LOO require training the learning algorithm

\footnotetext{
* Corresponding author.
} 
for every candidate kernel for several times, unavoidably bringing high computational burdens. For the sake of efficiency, some approximate CV approaches are proposed, such as, generalized cross validation (GCV)[14], generalized approximate cross validation (GACV) [27] and Bouligand influence function cross validation (BIFCV) [18]. Minimizing theoretical estimate bounds of the expected error is an alternative to kernel selection. The commonly used theoretical estimates usually introduce some measures of complexity [3], such as VC dimension [26], Rademacher complexity [4], maximal discrepancy [3], radius-margin bound [6] and compression coefficient [19].

Approximate kernel selection is an emerging approach to alleviating the computational burdens of kernel selection for the large scale application by introducing kernel matrix approximation into the kernel selection domain [9]10]. As pointed out in [65], a kernel selection criterion is not required to be an unbiased estimate of the expected error, instead the primary requirement is merely for the minimum of the kernel selection criterion to provide a reliable indication of the minimum of the expected error in kernel parameter space. Therefore, we argue that it is sufficient to calculate an approximate criterion that can discriminate the optimal kernel from the candidates. Although the idea of approximate kernel selection has been successfully applied in model selection of the least squares support vector machine (LSSVM) [9]10], two theoretical problems are still open: how kernel matrix approximation impacts the kernel selection criterion and whether this impact can be ignored for large enough examples.

In this paper, we define the notion of approximate consistency for kernel matrix approximation algorithm to tackle the theoretical problems and establish the preliminary foundations of approximate kernel selection. By analyzing the approximate consistency of different kernel matrix approximation algorithms, we can answer the question that, under what conditions, and how, the approximate kernel selection criterion converges to the accurate one. It is worth noting that the approximate consistency is defined for kernel matrix approximation algorithms and different from the classical "consistency", which is defined for learning algorithms. For two kernel selection criteria, we analyze the approximate consistency of two typical kernel matrix approximation algorithms. The results demonstrate the appositeness of kernel matrix approximation for kernel selection in a hierarchical structure. Empirical studies are also conducted to verify our theoretical findings on benchmark and synthetic data.

The rest of this paper is organized as follows. In Section 2, we introduce related work and contributions of this paper. In Section 3, we define two kernel selection criteria and simply demonstrate the approximate kernel selection scheme. Section 4 gives the definition of approximate consistency and further analyzes the approximate consistency of several kernel matrix approximation algorithms. We empirically study the approximate consistency in Section 5. Finally, we conclude in Section 6.

\section{Related Work and Contributions}

Kernel matrix approximation is an effective tool for reducing the computational burdens of kernel based learning. In order to achieve linear complexity in $l$, where $l$ is the number of examples, approximations from subsets of columns are considered: Nyström method [29], modified Nyström method [28], sparse greedy approximations [22] or 
incomplete Cholesky decomposition [12]. These methods are all low-rank approximations and have time complexity $O\left(p^{2} l\right)$ for an approximation of rank $p$. Constructing multilevel circulant matrix (MCM) to approximate kernel matrix is another effective strategy [24]239], which allows the multi-dimensional fast Fourier transform (FFT) to be utilized in solving learning algorithms with complexity of $O(l \log (l))$.

Column sampling and MCM approximation have been theoretically analyzed a lot $\left[\begin{array}{l|l|l|l|l|l|l}17 & 28 & 13 & 24 & 9\end{array}\right.$. However, the analysis provides a bound on the matrix approximation error for an appropriate norm (typically spectral, Frobenius and trace norm), but this is independent of specific learning problem and can not reveal the influence of kernel matrix approximation on learning. Recent literatures [7/30,16,29] measure the influence of kernel matrix approximation on hypothesis, but none of them reveal the influence of kernel matrix approximation on kernel selection criterion. Approximate consistency defined in this paper, for the first time, is to measure the difference between the accurate criterion calculated by original kernel matrix and the approximate criterion calculated by approximate kernel matrix, and further show the convergence of the difference for large enough examples.

The approach of approximate model selection was first proposed in [9], in which MCM approximation is adopted. Later, an extension to Nyström approximation for approximate model selection was proposed in [10]. Approximate model selection is a promising topic, especially for large scale applications. However, two fundamental questions require answering: how different kernel matrix approximation algorithms influence the kernel selection criterion and whether the approximate criterion converges to the accurate one. This paper provides answers to these questions.

\section{Approximate Kernel Selection}

In this section, we first present two kernel selection criteria and then give a brief introduction of approximate kernel selection.

We use $\mathcal{X}$ to denote the input space and $\mathcal{Y}$ the output domain. Usually we will have $\mathcal{X} \subseteq \mathbb{R}^{d}, \mathcal{Y}=\{-1,1\}$ for binary classification and $\mathcal{Y}=\mathbb{R}$ for regression. We assume $|y| \leq M$ for any $y \in \mathcal{Y}$, where $M$ is a constant. The training set is denoted by $\mathcal{S}=\left\{\left(\boldsymbol{x}_{1}, y_{1}\right), \ldots,\left(\boldsymbol{x}_{l}, y_{l}\right)\right\} \in(\mathcal{X} \times \mathcal{Y})^{l}$. The kernel $\kappa$ considered in this paper is a function from $\mathcal{X} \times \mathcal{X}$ to the field $\mathbb{R}$ such that for any finite set of inputs $\left\{\boldsymbol{x}_{1}, \ldots, \boldsymbol{x}_{l}\right\} \subseteq \mathcal{X}$, the matrix $\mathbf{K}=\left[\kappa\left(\boldsymbol{x}_{i}, \boldsymbol{x}_{j}\right)\right]_{i, j=1}^{l}$ is symmetric and positive definite (SPD). $\mathbf{K}$ is the kernel matrix. The reproducing kernel Hilbert space (RKHS) $\mathcal{H}_{\kappa}$ associated with the kernel $\kappa$ can be defined as $\mathcal{H}_{\kappa}=\overline{\operatorname{span}}\{\kappa(\boldsymbol{x}, \cdot): \boldsymbol{x} \in \mathcal{X}\}$, and the inner product $\langle\cdot, \cdot\rangle_{\mathcal{H}_{\kappa}}$ on $\mathcal{H}_{\kappa}$ is determined by $\left\langle\kappa(\boldsymbol{x}, \cdot), \kappa\left(\boldsymbol{x}^{\prime}, \cdot\right)\right\rangle_{\mathcal{H}_{\kappa}}=\kappa\left(\boldsymbol{x}, \boldsymbol{x}^{\prime}\right)$ for $\boldsymbol{x}, \boldsymbol{x}^{\prime} \in \mathcal{X}$. We use $\|\mathbf{K}\|_{2},\|\mathbf{K}\|_{\mathrm{F}}$ and $\|\mathbf{K}\|_{*}$ to denote the spectral, Frobenius and trace norm of $\mathbf{K}$. We use $\lambda_{t}(\mathbf{K})$ for $t=1, \ldots, l$ to denote the eigenvalues of $\mathbf{K}$ in the descending order.

Now we present two kernel selection criteria, which are both derived from the error estimation. The first one is from the regularized empirical error functional

$$
\mathcal{R}(f)=\frac{1}{l} \sum_{i=1}^{l}\left(f\left(\boldsymbol{x}_{i}\right)-y_{i}\right)^{2}+\mu\|f\|_{\mathcal{H}_{\kappa}}^{2},
$$


where $\mu$ is the regularization parameter. We denote the target function as $f_{\kappa}$ and $f_{\kappa}=$ $\arg \min _{f \in \mathcal{H}_{\kappa}} \mathcal{R}(f)$. Using the representer theorem, $f_{\kappa}$ can be represented as $f_{\kappa}=$ $\sum_{i=1}^{l} \alpha_{i} \kappa\left(\boldsymbol{x}_{i}, \cdot\right)$ with $\boldsymbol{\alpha}=\left(\alpha_{1}, \ldots, \alpha_{l}\right)^{\mathrm{T}}=(\mathbf{K}+\mu l \mathbf{I})^{-1} \boldsymbol{y}$, where $\boldsymbol{y}=\left(y_{1} \ldots, y_{l}\right)^{\mathrm{T}}$ and $\mathbf{I}$ is the identity matrix. Denoting $\mathbf{K}+\mu l \mathbf{I}=\mathbf{K}_{\mu}$, we have $\left\|f_{\kappa}\right\|_{\mathcal{H}_{\kappa}}^{2}=\boldsymbol{\alpha}^{\mathrm{T}} \mathbf{K} \boldsymbol{\alpha}=$ $\boldsymbol{y}^{\mathrm{T}} \mathbf{K}_{\mu}^{-1} \mathbf{K K}_{\mu}^{-1} \boldsymbol{y}$. We use bold $\boldsymbol{f}_{\kappa}$ to denote $\left(f_{\kappa}\left(\boldsymbol{x}_{1}\right), \ldots, f_{\kappa}\left(\boldsymbol{x}_{l}\right)\right)^{\mathrm{T}}$ and hence $\boldsymbol{f}_{\kappa}=$ $\mathbf{K} \boldsymbol{\alpha}=\mathbf{K K}_{\mu}^{-1} \boldsymbol{y}$, which implies $\boldsymbol{f}_{\kappa}-\boldsymbol{y}=-\mu l \mathbf{K}_{\mu}^{-1} \boldsymbol{y}$. Therefore,

$$
\begin{aligned}
\mathcal{R}\left(f_{\kappa}\right) & =\frac{1}{l}\left(\boldsymbol{f}_{\kappa}-\boldsymbol{y}\right)^{\mathrm{T}}\left(\boldsymbol{f}_{\kappa}-\boldsymbol{y}\right)+\mu\left\|f_{\kappa}\right\|_{\mathcal{H}_{\kappa}}^{2} \\
& =\mu^{2} l \boldsymbol{y}^{\mathrm{T}} \mathbf{K}_{\mu}^{-1} \mathbf{K}_{\mu}^{-1} \boldsymbol{y}+\mu \boldsymbol{y}^{\mathrm{T}} \mathbf{K}_{\mu}^{-1} \mathbf{K} \mathbf{K}_{\mu}^{-1} \boldsymbol{y} \\
& =\mu \boldsymbol{y}^{\mathrm{T}} \mathbf{K}_{\mu}^{-1} \boldsymbol{y} .
\end{aligned}
$$

There is a bijection between the set of kernels on $\mathcal{X}$ and that of reproducing kernel Hilbert spaces (RKHSs) on $\mathcal{X}$. For different RKHSs $\mathcal{H}_{\kappa}$, we may obtain different target functions $f_{\kappa}$. Then from all target functions, we select the one making $\mathcal{R}\left(f_{\kappa}\right)$ the smallest and the corresponding kernel will be the optimal one. We denote

$$
\mathcal{C}_{1}(\mathbf{K})=\mathcal{R}\left(f_{\kappa}\right)=\mu \boldsymbol{y}^{\mathrm{T}} \mathbf{K}_{\mu}^{-1} \boldsymbol{y} .
$$

Supposing we are given a prescribed set of kernels $\mathcal{K}=\left\{\kappa_{1}, \ldots, \kappa_{n}\right\}$, we can find the optimal kernel as $\kappa^{*}=\arg \min _{\kappa \in \mathcal{K}} \mathcal{C}_{1}(\mathbf{K})$.

We further present another kernel selection criterion, which is derived by the biasvariance decomposition of in-sample prediction error estimation [2]. In most practical cases, the observed output $\boldsymbol{y}=\left(y_{1}, \ldots, y_{l}\right)^{\mathrm{T}}$ is corrupted by some noises. We assume $y_{i}=\dot{y}_{i}+\xi_{i}, 1 \leq i \leq l$, where $\dot{\boldsymbol{y}}=\left[\dot{y}_{1}, \ldots \dot{y}_{l}\right]^{\mathrm{T}}$ is the unknown true output and the noise vector $\boldsymbol{\xi}=\left[\xi_{1}, \ldots, \xi_{i}\right]^{\mathrm{T}}$ is a random vector with mean 0 and finite covariance matrix C. $\boldsymbol{f}_{\kappa}$ is a linear function of $\boldsymbol{y}$, which is an estimate of $\dot{\boldsymbol{y}}$. The expected prediction error of $\boldsymbol{f}_{\kappa}$ can be represented as

$$
\begin{aligned}
\frac{1}{l} \mathbb{E}_{\boldsymbol{\xi}}\left\|\boldsymbol{f}_{\kappa}-\dot{\boldsymbol{y}}\right\|^{2} & =\frac{1}{l}\left\|\mathbb{E}_{\boldsymbol{\xi}} \boldsymbol{f}_{\kappa}-\dot{\boldsymbol{y}}\right\|^{2}+\frac{1}{l} \operatorname{trace}\left(\operatorname{var}_{\boldsymbol{\xi}}\left(\boldsymbol{f}_{\kappa}\right)\right) \\
& =\frac{1}{l}\left\|\mathbf{K} \mathbf{K}_{\mu}^{-1} \dot{\boldsymbol{y}}-\dot{\boldsymbol{y}}\right\|^{2}+\frac{1}{l} \operatorname{trace}\left(\mathbf{C K}^{2} \mathbf{K}_{\mu}^{-2}\right) \\
& =\underbrace{\mu^{2} l \dot{\boldsymbol{y}}^{\mathrm{T}} \mathbf{K}_{\mu}^{-2} \dot{\boldsymbol{y}}}_{\operatorname{bias}(\mathbf{K})}+\underbrace{\frac{1}{l} \operatorname{trace}\left(\mathbf{C} \mathbf{K}^{2} \mathbf{K}_{\mu}^{-2}\right)}_{\text {variance }(\mathbf{K})} .
\end{aligned}
$$

For $\mathbf{C}=\sigma^{2} \mathbf{I}$ with $\sigma^{2}$ as variance, we denote

$$
\mathcal{C}_{2}(\mathbf{K})=\operatorname{bias}(\mathbf{K})+\operatorname{variance}(\mathbf{K})=\mu^{2} l \dot{\boldsymbol{y}}^{\mathrm{T}} \mathbf{K}_{\mu}^{-2} \dot{\boldsymbol{y}}+\frac{\sigma^{2}}{l} \operatorname{trace}\left(\mathbf{K}^{2} \mathbf{K}_{\mu}^{-2}\right) .
$$

Now we simply review approximate kernel selection [9[10]. Supposing we have the training data $\mathcal{S}$, a prescribed kernel set $\mathcal{K}$, a kernel selection criterion $\mathcal{C}(\mathbf{K})$ and a kernel matrix approximation algorithm $\mathcal{A}$, which takes the kernel matrix $\mathbf{K}$ as input and generate the approximate matrix $\tilde{\mathbf{K}}$, we can describe the approximate kernel selection scheme as in Algorithm 1 The optimal kernel is selected by minimizing $\mathcal{C}(\tilde{\mathbf{K}})$. 


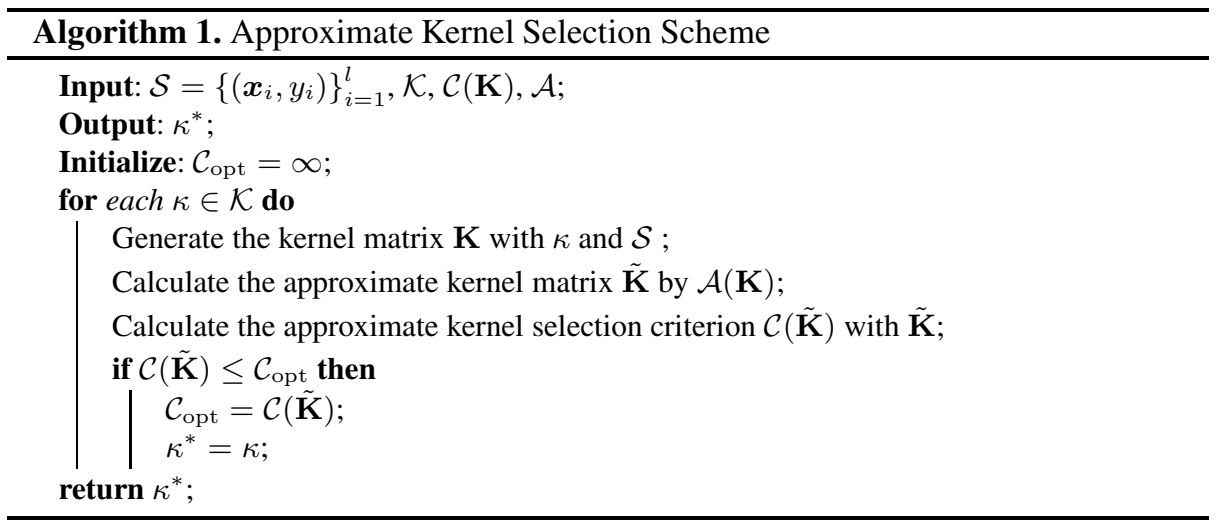

The computational cost for the criteria $\mathcal{C}_{1}(\mathbf{K})$ and $\mathcal{C}_{2}(\mathbf{K})$ is $O\left(l^{3}\right)$, which is prohibitive for large scale data. The computation of $\mathcal{C}(\tilde{\mathbf{K}})$ could be much more efficient than that of $\mathcal{C}(\mathbf{K})$ due to the specific structure of $\tilde{\mathbf{K}}$. For Nyström approximation [10] Woodbury formula could be used for calculating $\mathcal{C}(\tilde{\mathbf{K}})$ and for MCM approximation [9] multi-dimensional fast Fourier transform (FFT) could be used. The computational cost can even be reduced from $O\left(l^{3}\right)$ to $O(l \log (l))$ [9].

However, to demonstrate the rationality of approximate kernel selection, we need analyze what the difference between $\mathcal{C}(\mathbf{K})$ and $\mathcal{C}(\tilde{\mathbf{K}})$ is and whether this difference converges for large enough examples. In the next section, we will discuss these problems by defining the approximate consistency. At the end of this section, we introduce two typical kernel matrix approximation algorithms that will be discussed in this paper: Nyström approximation and MCM approximation.

The Nyström approximation generates a low rank approximation of $\mathbf{K}$ using a subset of the columns of $\mathbf{K}$. Suppose we randomly sample $c$ columns of $\mathbf{K} 1$ Let $\mathbf{C}$ denote the $l \times c$ matrix formed by theses columns. Let $\mathbf{D}$ be the $c \times c$ matrix consisting of the intersection of these $c$ columns with the corresponding $c$ rows of $\mathbf{K}$. The Nyström approximation matrix is $\tilde{\mathbf{K}}=\mathbf{C D}_{k}^{\dagger} \mathbf{C}^{\mathrm{T}} \approx \mathbf{K}$, where $\mathbf{D}_{k}$ is the optimal rank $k$ approximation to $\mathbf{D}$ and $\mathbf{D}_{k}^{\dagger}$ is the Moore-Penrose generalized inverse of $\mathbf{D}_{k}$. We further introduce the modified Nyström approximation [28], which shows tighter error bound than the standard Nyström method. The approximation matrix is $\tilde{\mathbf{K}}=\mathbf{C}\left(\mathbf{C}^{\dagger} \mathbf{K}\left(\mathbf{C}^{\dagger}\right)^{\mathrm{T}}\right) \mathbf{C}^{\mathrm{T}}$.

We now present the MCM approximation. We first briefly review the definition of $\operatorname{MCM} 2$ Let $\mathbb{N}$ denote the set of positive integers. For $m \in \mathbb{N}$, let $[m]=\{0,1, \ldots, m-$ $1\}$. For a fixed positive integer $p$, let $\boldsymbol{m}=\left(m_{0}, m_{1}, \ldots, m_{p-1}\right) \in \mathbb{N}^{p}$. We set $\Pi_{\boldsymbol{m}}=m_{0} m_{1} \ldots m_{p-1}$ and $[\boldsymbol{m}]=\left[m_{0}\right] \times\left[m_{1}\right] \times \cdots \times\left[m_{p-1}\right]$. A multilevel circulant matrix [25] is defined recursively. A 1-level circulant matrix is an ordinary circulant matrix. For any positive integer $s$, an $(s+1)$-level circulant matrix is a block circulant matrix whose blocks are $s$-level circulant matrices. According to [25], for $\boldsymbol{m} \in \mathbb{N}^{p}, \mathbf{A}_{\boldsymbol{m}}=\left[a_{\boldsymbol{i}, \boldsymbol{j}}: \boldsymbol{i}, \boldsymbol{j} \in[\boldsymbol{m}]\right]$ is a $p$-level circulant matrix if, for any $\boldsymbol{i}, \boldsymbol{j} \in[\boldsymbol{m}]$,

\footnotetext{
${ }^{1}$ Different sampling distributions have been considered [11|31|17|13].

2 Detailed definition can be seen in [25 9].
} 
$a_{\boldsymbol{i}, \boldsymbol{j}}=a_{i_{0}-j_{0}\left(\bmod m_{0}\right), \ldots, i_{p-1}-j_{p-1}\left(\bmod m_{p-1}\right)} . \mathbf{A}_{\boldsymbol{m}}$ is determined by its first column $a_{\boldsymbol{i}, \mathbf{0}}$ with $\mathbf{0}=(0, \ldots, 0) \in \mathbb{R}^{p}$. We write $\mathbf{A}_{\boldsymbol{m}}=\operatorname{circ}_{\boldsymbol{m}}\left[a_{\boldsymbol{i}}: \boldsymbol{i} \in[\boldsymbol{m}]\right]$, where $a_{\boldsymbol{i}}=a_{\boldsymbol{i}, \mathbf{0}}$, for $\boldsymbol{i} \in[\boldsymbol{m}]$. For the kernel function $\kappa$, we can construct MCM approximation of the kernel matrix $\mathbf{K}$ following the procedure given in Equation (12)-(14) of [9].

\section{Approximate Consistency}

In this section, we give the definition of approximate consistency and analyze the approximate consistency of Nyström approximation and MCM approximation.

Definition 1. Suppose we are given a kernel selection criterion $\mathcal{C}(\mathbf{K})$, which is a functional of the kernel matrix $\mathbf{K}$, and a kernel matrix approximation algorithm $\mathcal{A}$, which takes the kernel matrix $\mathbf{K}$ as input and generate the approximate matrix $\tilde{\mathbf{K}}$. We say the kernel matrix approximation algorithm $\mathcal{A}$ is of strong approximate consistency for the kernel selection criterion $\mathcal{C}(\mathbf{K})$, if

$$
|\mathcal{C}(\mathbf{K})-\mathcal{C}(\tilde{\mathbf{K}})| \leq \varepsilon(l),
$$

where $\lim _{l \rightarrow \infty} \varepsilon(l) \rightarrow 0$. We say $\mathcal{A}$ is of p-order approximate consistency for $\mathcal{C}(\mathbf{K})$ if

$$
|\mathcal{C}(\mathbf{K})-\mathcal{C}(\tilde{\mathbf{K}})| \leq \varepsilon(l),
$$

where $\lim _{l \rightarrow \infty} \varepsilon(l) / l^{p} \rightarrow 0$. There are two scenarios: if $\mathcal{A}$ is a deterministic algorithm, the approximate consistency is defined deterministically; if $\mathcal{A}$ is a stochastic algorithm, (6) or (7) is established under expectation or with high probability.

The approximate consistency reveals the convergence of the difference between the approximate kernel selection criterion and the accurate one. When kernel matrix approximation was applied in the kernel selection problem, the approximate consistency can be considered as a fundamental property of kernel matrix approximation algorithm to test its appositeness for kernel selection.

\subsection{Approximate Consistency of Nyström Approximation}

In this section we analyze the approximate consistency of Nyström approximation for the kernel selection criterion $\mathcal{C}_{1}(\mathbf{K})$.

Although there are many different versions of Nyström approximation, we concentrate on those with $(1+\epsilon)$ relative-error bounds, where $\epsilon$ does not depend on $l$. Two $(1+\epsilon)$ relative-error bounds have been reported for the standard Nyström [13] and modified Nyström method [28]. The bound for the standard Nyström method [13] states that for a failure probability $\delta \in(0,1]$ and an approximation factor $\epsilon \in(0,1]$,

$$
\|\mathbf{K}-\tilde{\mathbf{K}}\|_{*} \leq(1+\epsilon)\left\|\mathbf{K}-\mathbf{K}_{k}\right\|_{*}
$$

holds with probability at least $0.6-\delta$. The bound for the modified Nyström method [28] states that,

$$
\mathbb{E}\left(\|\mathbf{K}-\tilde{\mathbf{K}}\|_{\mathrm{F}}\right) \leq(1+\epsilon)\left\|\mathbf{K}-\mathbf{K}_{k}\right\|_{\mathrm{F}} .
$$


Before stating the main theorem of this section, we introduce two assumptions.

Assumption 1. For $\rho \in(0,1 / 2)$ and the rank parameter $k \leq c \ll l, \lambda_{k}(\mathbf{K})=\Omega\left(l / c^{\rho}\right)$ and $\lambda_{k+1}(\mathbf{K})=O\left(l / c^{1-\rho}\right)$, where $\rho$ is to characterize the eigengap.

Assumption 2. We always assume that the rank parameter $k$ is a constant and the sampling size $c$ is a small ratio $r$ of $l$.

Assumption 1 states the large eigengap in the spectrum of kernel matrix $\mathbf{K}$, i.e., the first few eigenvalues of the full kernel matrix are much larger than the remaining eigenvalues. This assumption has been adopted in [30 16] and empirically tested in [30]. Assumption 2 is one of common settings for Nyström approximation.

The following theorem shows the approximate consistency of the standard Nyström approximation using leverage score sampling [13]. The proof is given in Appendix A.

Theorem 1. For the kernel selection criterion $\mathcal{C}_{1}(\mathbf{K})$ defined in (3), if Assumption 1 and 2 hold, we have for $\delta \in(0,1]$ and $\epsilon \in(0,1]$,

$$
\left|\mathcal{C}_{1}(\mathbf{K})-\mathcal{C}_{1}(\tilde{\mathbf{K}})\right| \leq \varepsilon(l)
$$

holds with probability at least $0.6-\delta$, where $\tilde{\mathbf{K}}$ is produced by the standard Nyström approximation using leverage score sampling, $\varepsilon(l)=\frac{\tau M^{2}(1+\epsilon)}{\mu r^{1-\rho} l^{1-\rho}}(l-k)$ for some constant $\tau$ and $\lim _{l \rightarrow \infty} \varepsilon(l) / l^{\frac{1}{2}} \rightarrow 0$.

Theorem 1 demonstrates the $\frac{1}{2}$-order approximate consistency of the standard Nyström approximation for $\mathcal{C}_{1}(\mathbf{K})$. The strong approximate consistency has not been established. This is because the trace norm bound shown in (8), which is, to the best of our knowledge, the tightest bound for the standard Nyström approximation, is still not tight enough. If $(1+\epsilon)$ relative-error bound for spectral norm can be proved, we can derive the strong approximate consistency.

The following theorem shows the approximate consistency of the modified Nyström approximation [28]. The proof can be seen in Appendix A.

Theorem 2. For the kernel selection criterion $\mathcal{C}_{1}(\mathbf{K})$ defined in (3), if Assumption 1 and 2 hold, we have

$$
\mathbb{E}\left(\left|\mathcal{C}_{1}(\mathbf{K})-\mathcal{C}_{1}(\tilde{\mathbf{K}})\right|\right) \leq \varepsilon(l)
$$

where $\tilde{\mathbf{K}}$ is produced by the modified Nyström approximation, $\varepsilon(l)=\frac{\tau M^{2}(1+\epsilon)}{\mu r^{1-\rho} l^{1-\rho}} \sqrt{l-k}$ for some constant $\tau$ and $\lim _{l \rightarrow \infty} \varepsilon(l) \rightarrow 0$.

Theorem 2 demonstrates the strong approximate consistency of the modified Nyström approximation for $\mathcal{C}_{1}(\mathbf{K})$.

\subsection{Approximate Consistency of MCM Approximation}

In this section, we analyze the approximate consistency of MCM approximation for the criteria $\mathcal{C}_{1}(\mathbf{K})$ and $\mathcal{C}_{2}(\mathbf{K})$.

We use $\mathbf{U}_{\boldsymbol{m}}$ to denote the MCM that approximates the kernel matrix $\mathbf{K}$. To facilitate the analysis, we will rewrite the kernel matrix $\mathbf{K}$ in multilevel notation. For a given 
$\boldsymbol{m} \in \mathbb{N}^{p}$, we assume the number of elements in $\mathcal{S}$ is $\Pi_{\boldsymbol{m}}$, that is, $|\mathcal{S}|=l=\Pi_{\boldsymbol{m}}$. We relabel the elements in $\mathcal{S}$ using multi-index, $\mathcal{S}=\left\{\left(\boldsymbol{x}_{\boldsymbol{i}}, y_{\boldsymbol{i}}\right): \boldsymbol{i} \in[\boldsymbol{m}]\right\}$. In this notation, we rewrite $\mathbf{K}$ as $\mathbf{K}_{\boldsymbol{m}}=\left[K\left(\left\|\boldsymbol{x}_{\boldsymbol{i}}-\boldsymbol{x}_{\boldsymbol{j}}\right\|_{2}\right): \boldsymbol{i}, \boldsymbol{j} \in[\boldsymbol{m}]\right]^{3}$

The following theorem demonstrates the strong approximate consistency of MCM approximation for the criterion $\mathcal{C}_{1}(\mathbf{K})$. The proof is provided in Appendix B.

Theorem 3. If the following assumptions:

(H1) there exist positive constants $c_{0}$ and $\beta$ such that $|K(s)-K(t)| \leq c_{0}|s-t|^{\beta}$ for $s, t \in \mathbb{R}$;

(H2) there exists a positive constant $h$ such that $h_{\boldsymbol{m}, j} \geq h$ for $\boldsymbol{m} \in \mathbb{N}^{p}$ and $j \in[p]$;

(H3) there exist positive constants $\lambda_{1}$ and $c_{1}$ such that $|K(s)| \leq c_{1} \mathrm{e}^{-\lambda_{1}|s|}$ for $s \in \mathbb{R}$;

(H4) there exist positive constants $\lambda_{2}$ and $c_{2}$ such that for any $\boldsymbol{m} \in \mathbb{N}^{p}$, $\boldsymbol{i}, \boldsymbol{j} \in[\boldsymbol{m}], \quad\left|\left\|x_{\boldsymbol{i}}-x_{\boldsymbol{j}}\right\|_{2}-\left\|\left[\left(i_{s}-j_{s}\right) h_{\boldsymbol{m}, s}: s \in[p]\right]\right\|_{2}\right| \leq$ $c_{2} \sum_{s \in[p]}\left(\mathrm{e}^{-\lambda_{2} \delta_{m_{s}}\left(i_{s}\right)}+\mathrm{e}^{-\lambda_{2} \delta_{m_{s}}\left(j_{s}\right)}\right)$, where $\delta_{m}(j)=\frac{m}{2}-\left|\frac{m}{2}-j\right|$ for $m \in \mathbb{N}$ and $j \in[m]$;

hold and in addition, there exist positive constants $c_{3}$ and $r_{1}$ such that for any $\boldsymbol{m} \in \mathbb{N}^{p}$ and $\boldsymbol{i} \in[\boldsymbol{m}],\left|y_{\boldsymbol{i}}\right| \leq c_{3} \mathrm{e}^{-r_{1} \nu_{\boldsymbol{m}}(\boldsymbol{i})}$, where $\nu_{\boldsymbol{m}}(\boldsymbol{i})=\left\|\frac{\boldsymbol{m}}{2}-\boldsymbol{i}\right\|_{2}$, then we have

$$
\lim _{m \rightarrow \infty}\left|\mathcal{C}_{1}\left(\mathbf{K}_{\boldsymbol{m}}\right)-\mathcal{C}_{1}\left(\mathbf{U}_{\boldsymbol{m}}\right)\right|=0,
$$

where $\boldsymbol{m} \rightarrow \infty$ means all of its components go to infinity.

For the criterion $\mathcal{C}_{2}(\mathbf{K})$, we first give the following theorem. The detailed proof can be seen in Appendix B.

Theorem 4. If the assumptions (H1), (H2), (H3) and (H4) in Theorem 3 hold, then there exists a positive constant $c$ such that

$$
\left|\operatorname{variance}\left(\mathbf{K}_{\boldsymbol{m}}\right)-\operatorname{variance}\left(\mathbf{U}_{\boldsymbol{m}}\right)\right| \leq c \sigma^{2}\left(m_{\min }\right)^{-1},
$$

where $m_{\min }=\min \left\{m_{s}: s \in[p]\right\}$. If in addition, there exist positive constants $c_{3}$ and $r_{1}$ such that for any $\boldsymbol{m} \in \mathbb{N}^{p}$ and $\boldsymbol{i} \in[\boldsymbol{m}],\left|\dot{y}_{\boldsymbol{i}}\right| \leq c_{3} \mathrm{e}^{-r_{1} \nu_{\boldsymbol{m}}(\boldsymbol{i})}$, where $\nu_{\boldsymbol{m}}(\boldsymbol{i})=$ $\left\|\frac{m}{2}-i\right\|_{2}$, then there exist positive constants $c$ and $r$ such that for any $\boldsymbol{m} \in \mathbb{N}^{p}$

$$
\left|\operatorname{bias}\left(\mathbf{K}_{\boldsymbol{m}}\right)-\operatorname{bias}\left(\mathbf{U}_{\boldsymbol{m}}\right)\right| \leq c \mu^{2} \Pi_{\boldsymbol{m}}^{3 / 2} \mathrm{e}^{-r m_{\min }} .
$$

By Theorem 4, we can obtain the following theorem.

Theorem 5. If the assumptions (H1), (H2), (H3) and (H4) in Theorem 3 hold, we have

$$
\lim _{\boldsymbol{m} \rightarrow \infty}\left|\mathcal{C}_{2}\left(\mathbf{K}_{\boldsymbol{m}}\right)-\mathcal{C}_{2}\left(\mathbf{U}_{\boldsymbol{m}}\right)\right|=0 .
$$

Theorem 5 shows the strong approximate consistency of MCM approximation for the criterion $\mathcal{C}_{2}(\mathbf{K})$.

${ }^{3}$ There exists a real-valued function $K \in L^{1}(\mathbb{R})$ on $\mathcal{X}$ such that $K\left(\left\|\boldsymbol{x}-\boldsymbol{x}^{\prime}\right\|_{2}\right)=\kappa\left(\boldsymbol{x}, \boldsymbol{x}^{\prime}\right)$ for all $\boldsymbol{x}, \boldsymbol{x}^{\prime} \in \mathcal{X}$. 


\section{Empirical Studies}

In this section, we empirically study the approximate consistency of different kernel matrix approximation algorithms. We compare 6 approximation algorithms, including optimal rank $k$ approximation (OptApp), Nyström approximation with uniform sampling (Uniform) [17], column norm based sampling (ColNorm) [11], and leverage score based sampling (Leverage) [13], modified Nyström approximation (Modified) $[\sqrt{4}[$ and MCM approximation (MCM) [9].

We set the rank parameter $k=20$ and the sampling size $c=0.2 l$. To avoid the randomness, we run all Nyström methods 20 times. We use Gaussian kernels $\kappa\left(\boldsymbol{x}, \boldsymbol{x}^{\prime}\right)=\exp \left(-\gamma\left\|\boldsymbol{x}-\boldsymbol{x}^{\prime}\right\|_{2}^{2}\right)$ with different width $\gamma$ as our candidate kernel set $\mathcal{K}$. This paper does not focus on tuning the regularization parameter $\mu$, so we just set $\mu=0.005$. Since the regularized kernel matrix $\mathbf{K}_{\mu}=\mathbf{K}+\mu l \mathbf{I}, \mu=0.005$ is not too small.

We conduct experiments on benchmark and synthetic data. The benchmark data sets are 12 public available data sets from UCI repository 5 and LIBSVM Data 6 data sets for classification and 5 data sets for regression. To evaluate the evolution of the approximate consistency as the number of examples increases, we also generate the synthetic data. The target function is

$$
f(\boldsymbol{x})=\frac{1}{10}\left(\|\boldsymbol{x}\|_{2}+2 \mathrm{e}^{-8\left(\frac{4}{3} \pi-\|\boldsymbol{x}\|_{2}\right)^{2}}-2 \mathrm{e}^{-8\left(\frac{1}{2} \pi-\|\boldsymbol{x}\|_{2}\right)^{2}}-\mathrm{e}^{-8\left(\frac{3}{2} \pi-\|\boldsymbol{x}\|_{2}\right)^{2}}\right) .
$$

The points are $\left\{\left(\boldsymbol{x}_{\boldsymbol{j}}, y_{\boldsymbol{j}}\right), \boldsymbol{j} \in[\boldsymbol{m}]\right\} \in \mathbb{R}^{2} \times \mathbb{R}$ for $\boldsymbol{m}=(10,10),(20,20),(30,30)$. The sampled inputs $\boldsymbol{x}_{\boldsymbol{j}}$ is centered at 0 with fixed difference of any two successive numbers 0.1 , and $y_{\boldsymbol{j}}=f\left(\boldsymbol{x}_{\boldsymbol{j}}\right)+\xi$, where the noise $\xi$ is normally distributed with mean 0 and standard deviation 0.01 .

For each $\gamma$, we generate the kernel matrix $\mathbf{K}$ and then use different approximation algorithms to produce the approximate kernel matrices $\tilde{\mathbf{K}}$. We compare the values of $\mathcal{C}(\mathbf{K})$ and $\mathcal{C}(\tilde{\mathbf{K}})$. The results for benchmark dat: 7 are shown in Fig. 1 and Fig. 2] We can find that for most data sets the curves of the accurate and approximate criteria are close, and for the rest of data sets, although the values of the criteria are different, the lowest points of the curves are close, which means that the optimal kernels selected by minimizing the accurate and approximate criteria are close. Modified Nyström approximation shows better approximate consistency than the standard Nyström approximation, which is in accord with the theoretical results. The results for synthetic data are given in Fig. 3 to demonstrate the evolution of the approximate consistency as the number of examples increases. We can find that the more the number of examples is, the closer the curves of accurate and approximate criteria are.

\footnotetext{
${ }^{4}$ We only adopt uniform sampling for modified Nyström approximation.

5 http://www.ics.uci.edu/ mlearn/MLRepository.html

6 http://www.csie.ntu.edu.tw/ cjlin/libsvm

7 To satisfy the assumption (H4) in Theorem 3 we only conduct experiments on synthetic data for MCM approximation.
} 

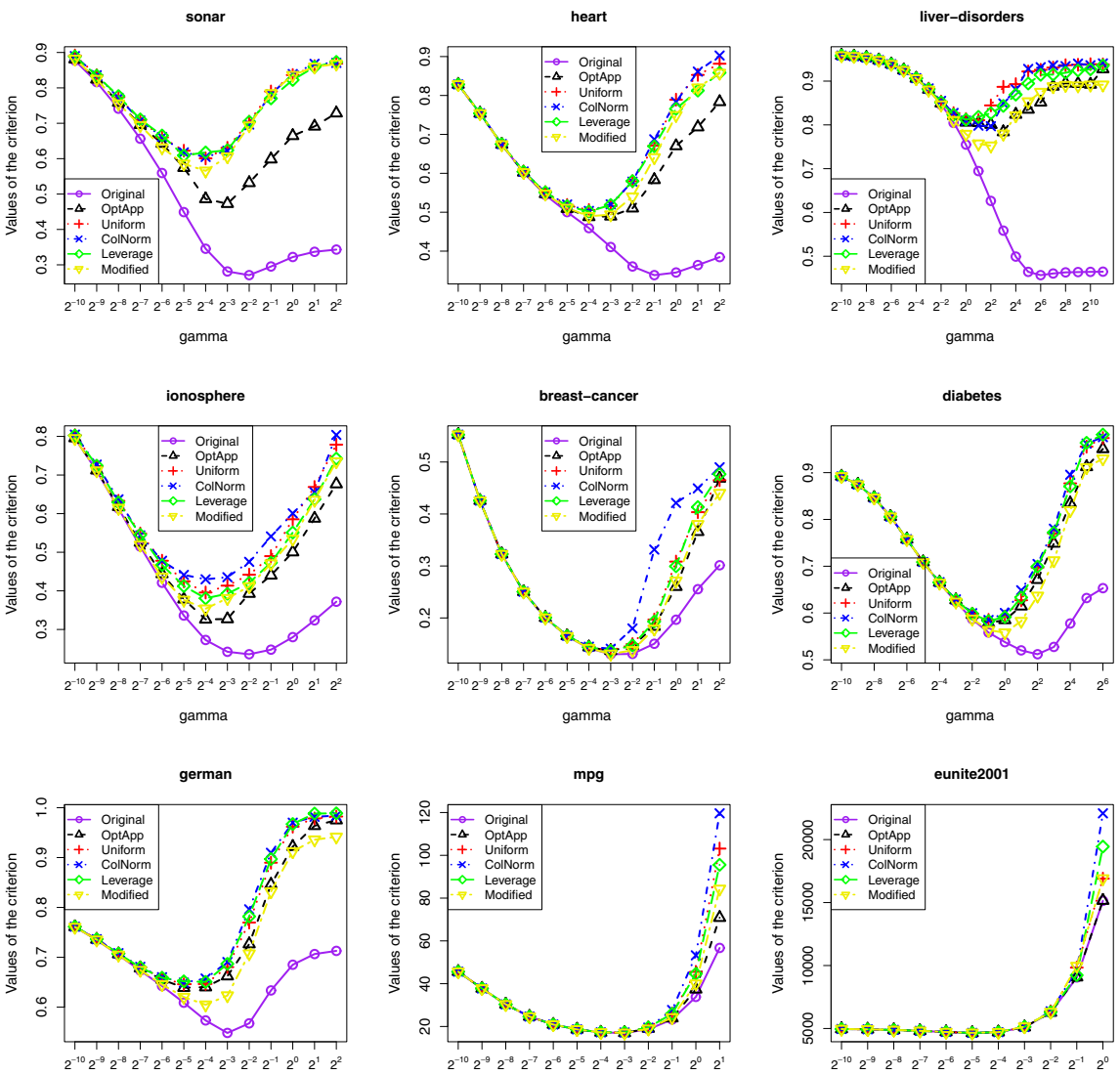

gamma

gamma
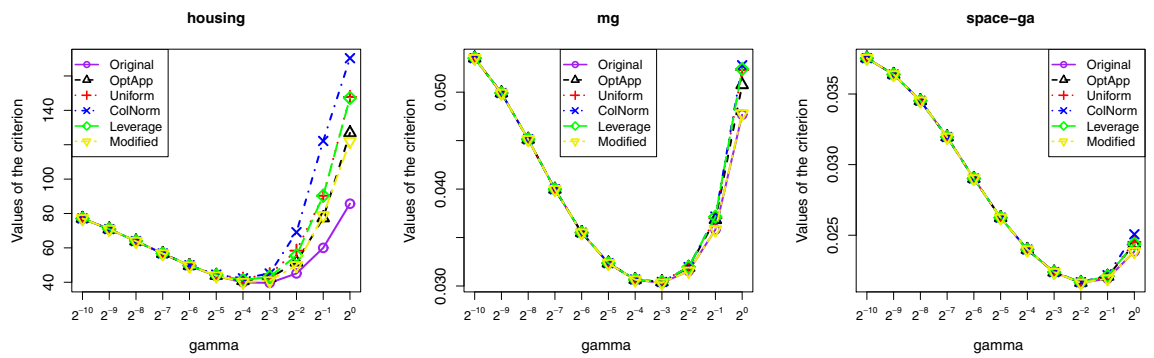

Fig. 1. Approximate consistency of different kernel matrix approximation algorithms for $\mathcal{C}_{1}(\mathbf{K})$ 

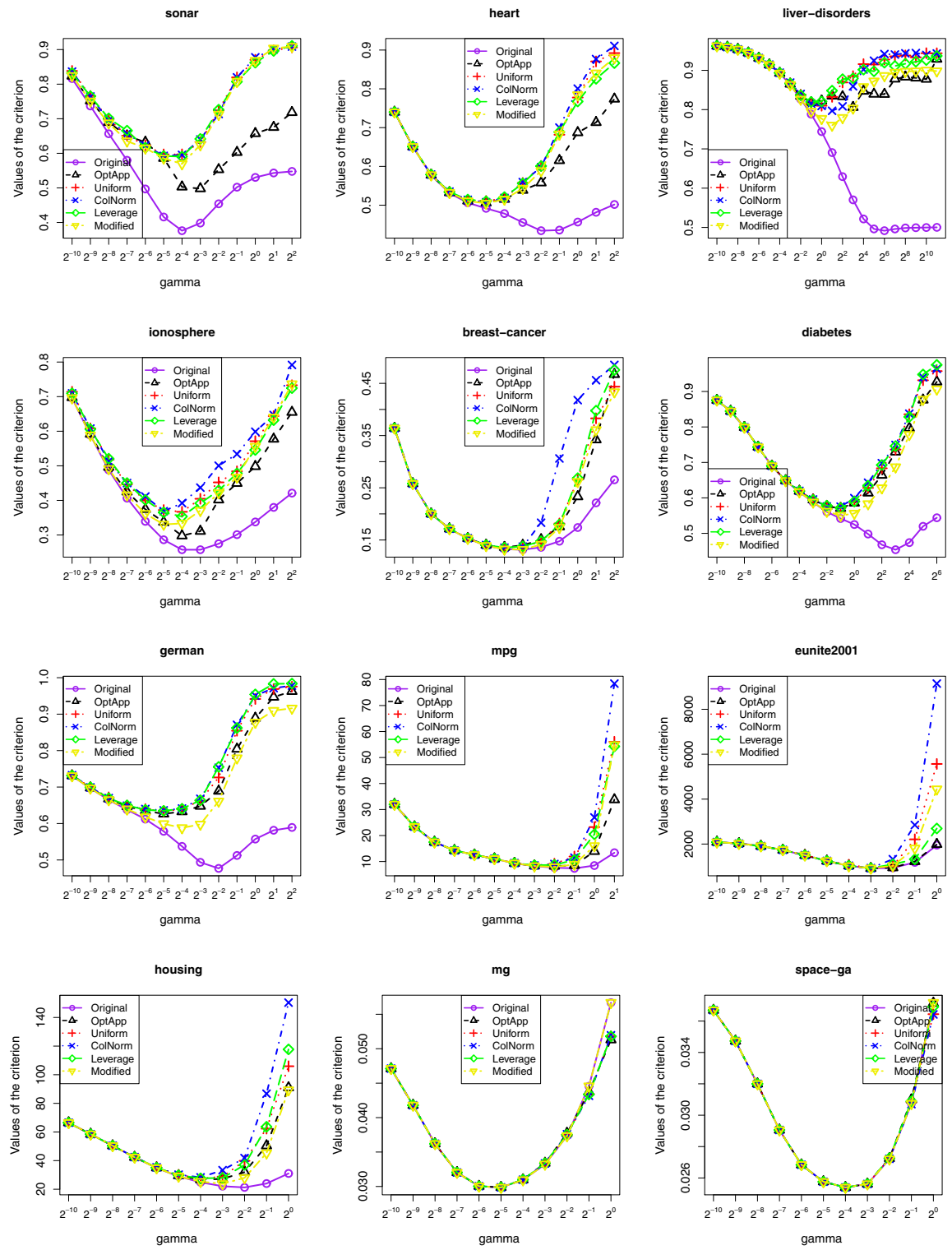

Fig. 2. Approximate consistency of different kernel matrix approximation algorithms for $\mathcal{C}_{2}(\mathbf{K})$ 

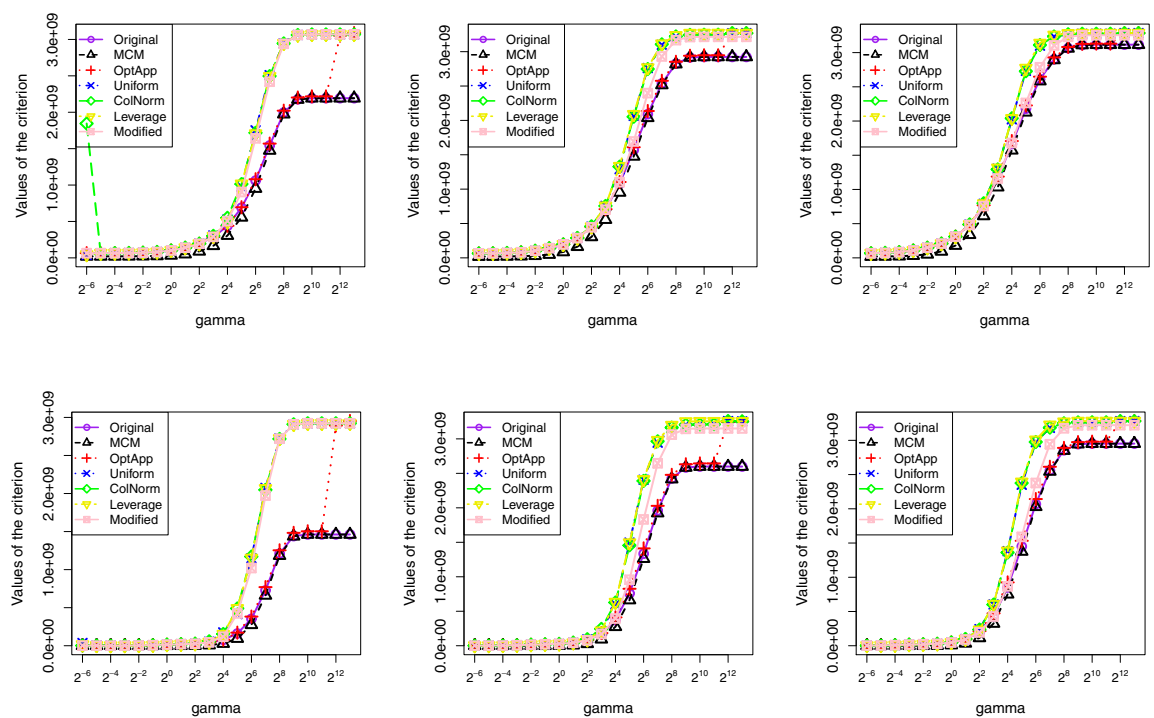

Fig. 3. Evolution of approximate consistency as the number of examples increases. The top 3 subfigures are for $\mathcal{C}_{1}(\mathbf{K})$ with $\boldsymbol{m}=(10,10),(20,20),(30,30)$ and the bottom 3 are for $\mathcal{C}_{2}(\mathbf{K})$.

\section{Conclusion}

In this paper, we defined the notion of approximate consistency for kernel matrix approximation algorithms, which tackles the theoretical problems faced by approximate kernel selection and establishes the preliminary foundations of approximate kernel selection. When kernel matrix approximation was applied in the kernel selection problem, approximate consistency can be considered as a fundamental property of kernel matrix approximation algorithm to test its appositeness for kernel selection. We have theoretically and empirically studied the approximate consistency of different kernel matrix approximation algorithms. To complete the foundations of approximate kernel selection, we will give the notion of hypothesis consistency, that is, the consistency of approximate optimal hypothesis and accurate optimal hypothesis, which are respectively learned using approximate and accurate optimal selected kernels, for future work.

Acknowledgments. The work is supported in part by the National Natural Science Foundation of China under grant No. 61170019.

\section{Appendix A: Proof of Theorem 1 and Theorem 2}

The proofs given in this section are partly based on the results in [7].

Let $\tilde{\mathbf{K}}=\mathcal{A}(\mathbf{K})$ be the produced approximate kernel matrix. We need to bound

$$
\begin{aligned}
\mathcal{C}_{1}(\mathbf{K})-\mathcal{C}_{1}(\tilde{\mathbf{K}}) & =\mu \boldsymbol{y}^{\mathrm{T}}(\mathbf{K}+\mu l \mathbf{I})^{-1} \boldsymbol{y}-\mu \boldsymbol{y}^{\mathrm{T}}(\tilde{\mathbf{K}}+\mu l \mathbf{I})^{-1} \boldsymbol{y} \\
& =-\mu \boldsymbol{y}^{\mathrm{T}}\left[(\mathbf{K}+\mu l \mathbf{I})^{-1}(\mathbf{K}-\tilde{\mathbf{K}})(\tilde{\mathbf{K}}+\mu l \mathbf{I})^{-1}\right] \boldsymbol{y},
\end{aligned}
$$


where the second equality follows that, for any invertible matrices $\mathbf{A}, \mathbf{B}$, the equality $\mathbf{A}^{-1}-\mathbf{B}^{-1}=-\mathbf{A}^{-1}(\mathbf{A}-\mathbf{B}) \mathbf{B}^{-1}$ holds. Then

$$
\begin{aligned}
\left|\mathcal{C}_{1}(\mathbf{K})-\mathcal{C}_{1}(\tilde{\mathbf{K}})\right| & \leq \mu\left\|\boldsymbol{y}^{\mathrm{T}}\right\|\left\|(\mathbf{K}+\mu l \mathbf{I})^{-1}\right\|_{2}\|\mathbf{K}-\tilde{\mathbf{K}}\|_{2}\left\|(\tilde{\mathbf{K}}+\mu l \mathbf{I})^{-1}\right\|_{2}\|\boldsymbol{y}\| \\
& \leq \frac{\mu\left\|\boldsymbol{y}^{\mathrm{T}}\right\|\|\mathbf{K}-\tilde{\mathbf{K}}\|_{2}\|\boldsymbol{y}\|}{\lambda_{\min }(\mathbf{K}+\mu l \mathbf{I}) \lambda_{\min }(\tilde{\mathbf{K}}+\mu l \mathbf{I})} \\
& \leq \frac{1}{\mu l^{2}}\left\|\boldsymbol{y}^{\mathrm{T}}\right\|\|\mathbf{K}-\tilde{\mathbf{K}}\|_{2}\|\boldsymbol{y}\|,
\end{aligned}
$$

where $\lambda_{\min }(\mathbf{M})$ denotes the smallest eigenvalue of $\mathbf{M}$. Since $\|\boldsymbol{y}\| \leq \sqrt{l} M$, we have

$$
\left|\mathcal{C}_{1}(\mathbf{K})-\mathcal{C}_{1}(\tilde{\mathbf{K}})\right| \leq \frac{M^{2}}{\mu l}\|\mathbf{K}-\tilde{\mathbf{K}}\|_{2}
$$

Now we give the proofs of Theorem 1 and Theorem 2

Proof (Theorem 1). Since $\|\mathbf{K}-\tilde{\mathbf{K}}\|_{2} \leq\|\mathbf{K}-\tilde{\mathbf{K}}\|_{*}$, substituting (8) into (19), we have for $\delta \in(0,1]$ and $\epsilon \in(0,1]$,

$$
\left|\mathcal{C}_{1}(\mathbf{K})-\mathcal{C}_{1}(\tilde{\mathbf{K}})\right| \leq \frac{M^{2}}{\mu l}(1+\epsilon)\left\|\mathbf{K}-\mathbf{K}_{k}\right\|_{*}
$$

holds with probability at least $0.6-\delta$. We know that $\left\|\mathbf{K}-\mathbf{K}_{k}\right\|_{*}=\sum_{t=k+1}^{l} \lambda_{t}(\mathbf{K})$. Therefore $\left\|\mathbf{K}-\mathbf{K}_{k}\right\|_{*} \leq(l-k) \lambda_{k+1}(\mathbf{K})$. Combining Assumption 1 and 2, we can obtain

$$
\left|\mathcal{C}_{1}(\mathbf{K})-\mathcal{C}_{1}(\tilde{\mathbf{K}})\right| \leq \varepsilon(l)
$$

with $\varepsilon(l)=O\left(\frac{M^{2}(1+\epsilon)}{\mu r^{1-\rho} l^{1-\rho}}(l-k)\right)$ and $\lim _{l \rightarrow \infty} \varepsilon(l) / l^{\frac{1}{2}} \rightarrow 0$, since $\rho<\frac{1}{2}$.

Proof (Theorem 2). Following the similar procedure as the proof of Theorem 1, we can obtain

$$
\mathbb{E}\left(\left|\mathcal{C}_{1}(\mathbf{K})-\mathcal{C}_{1}(\tilde{\mathbf{K}})\right|\right) \leq \varepsilon(l)
$$

with $\varepsilon(l)=O\left(\frac{M^{2}(1+\epsilon)}{\mu r^{1-\rho} l^{1-\rho}} \sqrt{l-k}\right)$ and $\lim _{l \rightarrow \infty} \varepsilon(l)=0$, since $\rho<\frac{1}{2}$.

\section{Appendix B: Proof of Theorem 3 and Theorem 4}

The proofs given in this section are mainly based on the results in [24[23].

We first introduce the "distances" of an entry in a multilevel matrix to its diagonal, to its upper right corner and to its lower left corner at each level [24]. For $t \in\{0,1\}, m \in$ $\mathbb{N}, i, j \in[m]$, we set $d_{m}(t, i, j):=t|i-j|+(1-t)(m-|i-j|-1)$ and for $\boldsymbol{t} \in\{0,1\}^{p}$, $\boldsymbol{m} \in \mathbb{N}^{p}, \boldsymbol{i}, \boldsymbol{j} \in[\boldsymbol{m}]$, let $\boldsymbol{d}_{\boldsymbol{m}}(\boldsymbol{t}, \boldsymbol{i}, \boldsymbol{j}):=\left[d_{m_{s}}\left(t_{s}, i_{s}, j_{s}\right): s \in[p]\right]$. For any $s \in[p]$, $d_{m_{s}}\left(1, i_{s}, j_{s}\right)$ is the distance of the entry at the position $\left(i_{s}, j_{s}\right)$ to the diagonal at level $s$ and $d_{m_{s}}\left(0, i_{s}, j_{s}\right)$ is the distance to the upper right and lower left corners at level $s$. For any $\boldsymbol{m} \in \mathbb{N}^{p}, \boldsymbol{i}, \boldsymbol{j} \in \mathbb{N}_{\boldsymbol{m}}$ and $r>0$, let $E_{r, p, \boldsymbol{m}}(\boldsymbol{i}, \boldsymbol{j}):=\sum_{\boldsymbol{t} \in\{0,1\}^{p}} \mathrm{e}^{-r\left\|\boldsymbol{d}_{\boldsymbol{m}}(\boldsymbol{t}, \boldsymbol{i}, \boldsymbol{j})\right\|_{2}}$. In what follows, we write $\mathcal{A}=\left\{\mathbf{A}_{\boldsymbol{m}}: \boldsymbol{m} \in \mathbb{N}^{p}\right\}, \mathcal{A}^{-1}=\left\{\mathbf{A}_{\boldsymbol{m}}^{-1}: \boldsymbol{m} \in \mathbb{N}^{p}\right\}$, $\mathcal{B}=\left\{\mathbf{B}_{\boldsymbol{m}}: \boldsymbol{m} \in \mathbb{N}^{p}\right\}$ and $\mathcal{A B}=\left\{\mathbf{A}_{\boldsymbol{m}} \mathbf{B}_{\boldsymbol{m}}: \boldsymbol{m} \in \mathbb{N}^{p}\right\}$.

We introduce two definitions about a class of matrices whose entries have an exponential decay property [24]. 
Definition 2. A sequence of positive definite matrices $\mathcal{A}$ belongs to $\mathcal{E}_{r}$ for a positive constant $r$ if it satisfies the following conditions: (i) there exists a positive constant $\kappa$ such that $\left\|\mathbf{A}_{\boldsymbol{m}}^{-1}\right\|_{2} \leq \kappa$ for any $\boldsymbol{m} \in \mathbb{N}^{p}$; (ii) there exists a positive constant $c$ such that for any $\boldsymbol{m} \in \mathbb{N}^{p}$ and $\boldsymbol{i}, \boldsymbol{j} \in[\boldsymbol{m}],\left|a_{\boldsymbol{i}, \boldsymbol{j}}\right| \leq c E_{r, p, \boldsymbol{m}}(\boldsymbol{i}, \boldsymbol{j})$.

Definition 3. We say $\mathcal{A}$ and $\mathcal{B}$ are asymptotically equivalent in $\mathcal{E}_{r}$ if $\mathcal{A}, \mathcal{B} \in \mathcal{E}_{r}$ and there exists a positive constant $c$ such that for any $\boldsymbol{m} \in \mathbb{N}^{p}$ and $\boldsymbol{i}, \boldsymbol{j} \in[\boldsymbol{m}], \mid a_{\boldsymbol{i}, \boldsymbol{j}}-$ $b_{\boldsymbol{i}, \boldsymbol{j}} \mid \leq c \sum_{s \in[p]}\left(\mathrm{e}^{-r \delta_{m_{s}}\left(i_{s}\right)}+\mathrm{e}^{-r \delta_{m_{s}}\left(j_{s}\right)}\right)$, where $\delta_{m}(j)=\frac{m}{2}-\left|\frac{m}{2}-j\right|$, for $m \in \mathbb{N}$, $j \in[m]$. We use the notation $\mathcal{A} \sim \mathcal{E}_{r} \mathcal{B}$.

We further introduce two propositions and a lemma [23].

Proposition 1. If $\mathcal{A}, \mathcal{B} \in \mathcal{E}_{r}$, then $\mathcal{A B} \in \mathcal{E}_{r_{0}}$ for any $r_{0}<r$.

Proposition 2. If $\mathcal{A} \sim \mathcal{E}_{r} \mathcal{B}$, then $\mathcal{A}^{-1} \sim_{\mathcal{E}_{r^{\prime}}} \mathcal{B}^{-1}$ for any $r^{\prime}<r$.

Lemma 1. If $\mathcal{A} \sim_{\mathcal{E}_{r}} \mathcal{B}$ and $\mathcal{D} \in \mathcal{E}_{r}$, then $\mathcal{D} \mathcal{A} \sim_{\mathcal{E}_{r_{1}}} \mathcal{D B}$ and $\mathcal{A D} \sim \mathcal{E}_{r_{1}} \mathcal{B D}$ for some $r_{1}<r$.

Using above results [23], we prove the following three propositions.

Proposition 3. If $\mathcal{A} \sim \mathcal{E}_{r} \mathcal{B}$, then $\mathcal{A}^{2} \sim \mathcal{E}_{r^{\prime}} \mathcal{B}^{2}$, for some $r^{\prime}<r$.

Proof. From Proposition 1, we have $\mathcal{A}^{2}=\mathcal{A} \mathcal{A}, \mathcal{B}^{2}=\mathcal{B B}$, and $\mathcal{A B}$ are in $\mathcal{E}_{r_{0}}$ for any $r_{0}<r$. From Lemma1, we can obtain $\mathcal{A}^{2} \sim_{\mathcal{E}_{r_{1}}} \mathcal{A B}$ for some $r_{1}<r$ and $\mathcal{A B} \sim_{\mathcal{E}_{r_{2}}} \mathcal{B}^{2}$ for some $r_{2}<r$. We take $r^{\prime}=\min \left\{r_{1}, r_{2}\right\}$. According to Definition 3 and triangular inequality, we have $\mathcal{A}^{2} \sim \mathcal{E}_{r^{\prime}} \mathcal{B}^{2}$.

Proposition 4. If $\mathcal{A} \sim \mathcal{E}_{r_{1}} \mathcal{B}$ and $\mathcal{C} \sim \mathcal{E}_{r_{2}} \mathcal{D}$, then $\mathcal{A C} \sim_{\mathcal{E}_{r_{0}}} \mathcal{B D}$, for some $r_{0}<$ $\min \left\{r_{1}, r_{2}\right\}$.

Proposition 5. If $\mathcal{A} \in \mathcal{E}_{r_{1}}$ and $\mathcal{B} \in \mathcal{E}_{r_{2}}$ then $\mathcal{A B} \in \mathcal{E}_{r_{0}}$ for any $r_{0}<\min \left\{r_{1}, r_{2}\right\}$.

The proof of Proposition 5 is similar to Proposition 1, Now we prove Proposition 4.

Proof (Proposition 4). Following Lemma 1 and Proposition 5, we have $\mathcal{A C} \sim \mathcal{E}_{r_{3}}$ BC for some $r_{3}<\min \left\{r_{1}, r_{2}\right\}$ and $\mathcal{B C} \sim \mathcal{E}_{r_{4}} \mathcal{B D}$ for some $r_{4}<\min \left\{r_{1}, r_{2}\right\}$. Therefore, $\mathcal{A C} \sim \mathcal{E}_{r_{0}} \mathcal{B D}$ for some $r_{0}=\min \left\{r_{3}, r_{4}\right\}<\min \left\{r_{1}, r_{2}\right\}$.

Now we prove Theorem 3 and Theorem 4

Proof (Theorem 3). We write $\mathcal{K}_{\mu}=\left\{\mathbf{K}_{\boldsymbol{m}}+\mu l \mathbf{I}_{\boldsymbol{m}}: \boldsymbol{m} \in \mathbb{N}^{p}\right\}$ and $\mathcal{U}_{\mu}=\left\{\mathbf{U}_{\boldsymbol{m}}+\mu l \mathbf{I}_{\boldsymbol{m}}\right.$ : $\left.\boldsymbol{m} \in \mathbb{N}^{p}\right\}$. Theorem 5.2.3 in [23] states that if the assumptions (H1), (H2), (H3) and (H4) in Theorem 3 hold, $\mathcal{K}_{\mu} \sim \mathcal{E}_{r} \mathcal{U}_{\mu}$, where $r=\min \left\{\lambda_{1} h, \lambda_{2} \beta\right\}$. Combining Theorem 5.2.3 in [23] with Theorem 4.2.2 in [23], we can obtain Theorem 3

Proof (Theorem 4). We denote $\mathcal{K}_{\mu}^{-q}=\left\{\left(\mathbf{K}_{\boldsymbol{m}}+\mu l \mathbf{I}_{\boldsymbol{m}}\right)^{-q}: \boldsymbol{m} \in \mathbb{N}^{p}\right\}$ and $\mathcal{U}_{\mu}^{-q}=$ $\left\{\left(\mathbf{U}_{\boldsymbol{m}}+\mu l \mathbf{I}_{\boldsymbol{m}}\right)^{-q}: \boldsymbol{m} \in \mathbb{N}^{p}\right\}$ for $q=1,2, \ldots$ According to Proposition 3 and Theorem 5.2.3 in [23], we have $\mathcal{K}_{\mu}^{-2} \sim \mathcal{E}_{r^{\prime}} \mathcal{U}_{\mu}^{-2}$, for some $r^{\prime}<r$. Based on Proposition 4.2.1 in [23], we have $\left|\operatorname{bias}\left(\mathbf{K}_{\boldsymbol{m}}\right)-\operatorname{bias}\left(\mathbf{U}_{\boldsymbol{m}}\right)\right| \leq c \mu^{2} \Pi_{\boldsymbol{m}}^{3 / 2} \mathrm{e}^{-r^{\prime} m_{\min }}$, which is the 
bound for bias term in Theorem 2. Since the kernel matrix considered in this paper is assumed to be SPD, the corresponding multilevel circulant matrix is also positive definite. We have the matrix sets $\mathcal{K}=\left\{\mathbf{K}_{\boldsymbol{m}}: \boldsymbol{m} \in \mathbb{N}^{p}\right\}$ and $\mathcal{U}=\left\{\mathbf{U}_{\boldsymbol{m}}: \boldsymbol{m} \in\right.$ $\left.\mathbb{N}^{p}\right\}$ are asymptotically equivalent. Therefore, according to Proposition 3 and 4 we have $\mathcal{K}^{2} \mathcal{K}_{\mu}^{-2} \sim_{\mathcal{E}_{r_{0}}} \mathcal{U}^{2} \mathcal{U}_{\mu}^{-2}$ for some $r_{0}<r^{\prime}$. By Proposition 4.3 .2 of [23], we can obtain $\left|\operatorname{trace}\left(\mathbf{K}_{\boldsymbol{m}}^{2}\left(\mathbf{K}_{\boldsymbol{m}}+\mu l \mathbf{I}_{\boldsymbol{m}}\right)^{-2}\right)-\operatorname{trace}\left(\mathbf{U}_{\boldsymbol{m}}^{2}\left(\mathbf{U}_{\boldsymbol{m}}+\mu l \mathbf{I}_{\boldsymbol{m}}\right)^{-2}\right)\right| \leq c \Pi_{\boldsymbol{m}}\left(m_{\min }\right)^{-1}$. Therefore, we have $\left|\operatorname{variance}\left(\mathbf{K}_{\boldsymbol{m}}\right)-\operatorname{variance}\left(\mathbf{U}_{\boldsymbol{m}}\right)\right| \leq c \sigma^{2}\left(m_{\min }\right)^{-1}$, which is the bound for variance term in Theorem 4

\section{References}

1. Alpaydin, E.: Introduction to Machine Learning. MIT Press, Cambridge (2004)

2. Bach, F.: Sharp analysis of low-rank kernel matrix approximations. In: Proceedings of the 26th Annual Conference on Learning Theory (COLT), pp. 185-209 (2013)

3. Bartlett, P.L., Boucheron, S., Lugosi, G.: Model selection and error estimation. Machine Learning 48(1-3), 85-113 (2002)

4. Bartlett, P., Mendelson, S.: Rademacher and Gaussian complexities: Risk bounds and structural results. Journal of Machine Learning Research 3, 463-482 (2002)

5. Cawley, G., Talbot, N.: On over-fitting in model selection and subsequent selection bias in performance evaluation. Journal of Machine Learning Research 11, 2079-2107 (2010)

6. Chapelle, O., Vapnik, V., Bousquet, O., Mukherjee, S.: Choosing multiple parameters for support vector machines. Machine Learning 46(1-3), 131-159 (2002)

7. Cortes, C., Mohri, M., Talwalkar, A.: On the impact of kernel approximation on learning accuracy. In: Proceedings of the 13th International Conference on Artificial Intelligence and Statistics (AISTATS), pp. 113-120 (2010)

8. De Vito, E., Caponnetto, A., Rosasco, L.: Model selection for regularized least-squares algorithm in learning theory. Foundations of Computational Mathematics 5(1), 59-85 (2005)

9. Ding, L.Z., Liao, S.Z.: Approximate model selection for large scale LSSVM. Journal of Machine Learning Research - Proceedings Track 20, 165-180 (2011)

10. Ding, L., Liao, S.: Nyström approximate model selection for LSSVM. In: Tan, P.-N., Chawla, S., Ho, C.K., Bailey, J. (eds.) PAKDD 2012, Part I. LNCS (LNAI), vol. 7301, pp. 282-293. Springer, Heidelberg (2012)

11. Drineas, P., Mahoney, M.W.: On the Nyström method for approximating a Gram matrix for improved kernel-based learning. Journal of Machine Learning Research 6, 2153-2175 (2005)

12. Fine, S., Scheinberg, K.: Efficient SVM training using low-rank kernel representations. Journal of Machine Learning Research 2, 243-264 (2002)

13. Gittens, A., Mahoney, M.W.: Revisiting the Nyström method for improved large-scale machine learning. In: Proceedings of the 30th International Conference on Machine Learning (ICML), pp. 567-575 (2013)

14. Golub, G.H., Heath, M., Wahba, G.: Generalized cross-validation as a method for choosing a good ridge parameter. Technometrics 21(2), 215-223 (1979)

15. Hastie, T., Rosset, S., Tibshirani, R., Zhu, J.: The entire regularization path for the support vector machine. Journal of Machine Learning Research 5, 1391-1415 (2004)

16. Jin, R., Yang, T.B., Mahdavi, M., Li, Y.F., Zhou, Z.H.: Improved bounds for the Nyström method with application to kernel classification. IEEE Transactions on Information Theory 5(10), 6939-6949 (2013)

17. Kumar, S., Mohri, M., Talwalkar, A.: Sampling methods for the Nyström method. Journal of Machine Learning Research 13, 981-1006 (2012) 
18. Liu, Y., Jiang, S., Liao, S.: Efficient approximation of cross-validation for kernel methods using Bouligand influence function. In: Proceedings of the 31st International Conference on Machine Learning (ICML), pp. 324-332 (2014)

19. Luxburg, U.V., Bousquet, O., Schölkopf, B.: A compression approach to support vector model selection. Journal of Machine Learning Research 5, 293-323 (2004)

20. Micchelli, C.A., Pontil, M.: Learning the kernel function via regularization. Journal of Machine Learning Research 6, 1099-1125 (2005)

21. Mitchell, T.M.: Machine Learning. McGraw Hill, New York (1997)

22. Smola, A.J., Schölkopf, B.: Sparse greedy matrix approximation for machine learning. In: Proceedings of the 17th International Conference on Machine Learning (ICML), pp. 911-918 (2000)

23. Song, G.H.: Approximation of kernel matrices in machine learning. Ph.D. thesis, Syracuse University, Syracuse, NY, USA (2010)

24. Song, G.H., Xu, Y.S.: Approximation of high-dimensional kernel matrices by multilevel circulant matrices. Journal of Complexity 26(4), 375-405 (2010)

25. Tyrtyshnikov, E.E.: A unifying approach to some old and new theorems on distribution and clustering. Linear Algebra and its Applications 232, 1-43 (1996)

26. Vapnik, V.: The Nature of Statistical Learning Theory. Springer, New York (1995)

27. Wahba, G., Lin, Y., Zhang, H.: GACV for support vector machines. In: Advances in Large Margin Classifiers. MIT Press, Cambridge (1999)

28. Wang, S.S., Zhang, Z.H.: Improving CUR matrix decomposition and the Nyström approximation via adaptive sampling. Journal of Machine Learning Research 14, 2729-2769 (2013)

29. Williams, C.K.I., Seeger, M.: Using the Nyström method to speed up kernel machines. In: Advances in Neural Information Processing Systems 13, pp. 682-688 (2001)

30. Yang, T.B., Li, Y.F., Mahdavi, M., Jin, R., Zhou, Z.H.: Nyström method vs random Fourier features: A theoretical and empirical comparison. In: Advances in Neural Information Processing Systems 24, pp. 1060-1068 (2012)

31. Zhang, K., Kwok, J.T.: Clustered Nyström method for large scale manifold learning and dimension reduction. IEEE Transactions on Neural Networks 21(10), 1576-1587 (2010) 